\title{
L'anemia del Gottardo
}

\author{
Luigi Belloni (Milano)
}

L'onore che mi dà la Società Svizzera di Storia della Medicina di portare alla celebrazione del suo cinquantenario l'augurio dei colleghi Italiani, accentua il mio ormai vecchio debito di gratitudine, sia vero il Paese che mi ha accolto e protetto in anni tristissimi per la mia Patria, sia verso la Società che, in quegli stessi anni, accogliendo benevolmente su «Gesnerus» il mio primo scritto di Storia della Medicina, ha non poco contribuito alla mia evoluzione verso questa disciplina. Arbitro del destino di quel mio scritto fu il prof. Hans Fischer, redattore di Gesnerus: e mi è grato oggi riprendere il discorso iniziato nel 1962 sulla «Festschrift» in suo onore ${ }^{1}$.

La scoperta dell'Ankylostoma duodenale, compiuta a Milano nel 1838, $\grave{\mathrm{e}}$, infatti, il primo presupposto per la soluzione dell'enigma patologico posto quarant'anni più tardi dai lavoratori addetti al traforo del San Gottardo, iniziato nel 1872 e inaugurato al traffico nel 1882.

In quel mio articolo non ho ignorato il problema, molto interessante per la storia critica della scienza, dei moventi che indussero Angelo Dubini (1813-1902), lo scopritore dell'Ankylostoma, a scagionare il verme dai gravi quadri anemici che, da finissimo anatomo-patologo, egli aveva costatato nei cadaveri dei soggetti che ne erano portatori.

Imperava allora l'anticontagionismo, come ha messo così bene in rilievo l'Ackerknecht ${ }^{2}$ : e il Dubini - benchè partigiano del contagio vivo per le malattie da Acari e da Miceti - preferì sottovalutare i reperti, umani ed elmintici, probativi per una attività patogena dell'Ankylostoma, e porre invece l'accento sui dati di fatto eloquenti in senso opposto, come il ritrovamento del verme «in venti cadaveri almeno di cento che si dissecano colla mira di ritrovarlo», nonchè la frequente osservazione di vermi liberi nel lume intestinale e avvolti nel muco. Si tratta di un fenomeno cadaverico

1 L. Belloni, La scoperta dell'Ankylostoma duodenale, Gesnerus 19 (1962) 101-118. Non riporto ora molte citazioni contenute in quell'articolo, ricordando, peraltro, l'esauriente Bibliography of Hookworm Disease (New York, Rockefeller Foundation, International Health Board, 1922).

2 E.H.Ackerknecht, Anticontagionism between 1821 and 1867, Bull. Hist. Med. 22 (1948) 562-593. 
oggi ben noto: quanto maggiore è il tempo intercorso fra la morte e l'autopsia, tanto più numerosi sono i vermi liberi nel lume intestinale.

Il reperto di vermi tenacemente ancorati al duodeno si impose, invece, in tutta la sua evidenza agli occhi di settori attivi in paesi tropicali, dopo che nel 1845 lo zoologo Karl-Theodor-Ernst von Siebold (1804-85) ${ }^{3}$ aveva contribuito a far conoscere l'Ankylostoma in base alla pubblicazione del Dubini, che risale all'aprile del $1843^{4}$.

Nel 1847 Franz Pruner (Pruner Bey, 1808-82) ${ }^{5}$ indicò la elevata frequenza dell'Ankylostoma nel corso delle sezioni cadaveriche praticate in Egitto: il parassita aderisce alla mucosa intestinale grazie agli uncini del suo apparato succhiatore e produce le ecchimosi. Wilhelm Griesinger (1817 a 1868$)^{6}$ e Theodor Bilharz (1825-62) ${ }^{7}$, trasferitisi in Egitto nel 1850, pro-

${ }^{3}$ K.Tн. E.v. SieboLd, Bericht über die Leistungen im Gebiete der Helminthologie während des Jahres 1843 und 1844, Archiv für Naturgeschichte 2 (1845) 202-255 (cfr. 220-221).

4 A. Dubini, Nuovo verme intestinale umano (Agchylostoma duodenale), costituente un sesto genere di Nematoidei proprii dell'uomo, Ann. universali med. 106 (1843) 5-13; Notizia di un nuovo verme degli intestini umani, costituente un settimo genere di Nematoidei proprii dell'uomo, Gazz. med. di Milano 2 (1843) 153-154.

5 F.Pruner, Die Krankheiten des Orient's vom Standpunkte der vergleichenden Nosologie betrachtet von Dr.F. Pruner, Erlangen 1847, pp. 244-245: «Selten öffnet man eine Leiche in Ägypten ohne Individuen von einer oder auch mehreren Arten zu finden. Der Ascaris lumbr., Ascaris und Oxyuris vermicularis und Trichocephalus dispar finden sich zu Haufen schon in den Gedärmen der Kinder. Unter den Erwachsenen sind es besonders die kachektischen, wassersüchtigen und skrofulösen Subjekte, welche außerdem an Anchylostoma duodenale im Zwölffingerdarme leiden, wo dieser Parasit seinen vierfächerigen Saugerüssel mit 40 Haken an die Schleimhaut heftet, wodurch er Ecchymosen veranlaßt.»

6 W. Griesinger, Klinische und anatomische Beobachtungen über die Krankheiten von Egypten, Archiv für physiologische Heilkunde a più puntate; cf. 13 (1854) 555-561 («Anchylostomenkrankheit und Chlorose»). Dello stesso Griesinger si veda inoltre la breve comunicazione qui citata alla nota 11 .

7 Tн.Bilharz, Ein Beitrag zur Helminthographia humana, aus brieflichen Mitteilungen des Dr. Bilharz in Cairo, nebst Bemerkungen von Professor C. Th.v. Siebold in Breslau, Z. wissensch. Zoologie 4 (1852) 53-76, tav. V. Vedi a p. 56: «Die Stelle, wo ein solcher Strongylus saß, ist durch eine linsengroße Ecchymose bezeichnet, in deren Mitte ein weißer Fleck von Stecknadelgröße bemerkbar. Dieser weiße Fleck ist in der Mitte durchbohrt von einem nadeldicken bis in das submucöse Bindegewebe dringenden Loche. Manchmal zeigt die Schleimhaut flache Erhabenheiten von Linsengröße und livid braunrother Farbe, welche eine zwischen Tunica mucosa und muscularis im Bindegewebe befindliche, mit Blut gefüllte Höhle und darin zusammengeringelt den lebenden von Blut voll gesogenen Wurm (bald ein Männchen, bald ein Weibchen) enthalten.» 
cedettero oltre: l'enterorragia che, in un caso sezionato il 17 aprile 1852 , affiancava le ecchimosi, accentuò l'analogia con l'azione della sanguisuga. La c.d. clorosi egiziana, una grave e fatale malattia cachettizzante largamente endemica in Egitto, è concepibile quale Anchylostomenkrankheit.

L'Ankylostoma egiziano si àncora solidamente alla mucosa duodenale, e succhia abbondante sangue, anemizzando progressivamente l'individuo infestato fino alla morte (anemia perniciosa progressiva); laddove l'Ankylostoma lombardo è molto meno pericoloso e preferisce abitualmente rimanere libero nel lume intestinale, così come lo aveva ritrovato il Dubini. Questa è sostanzialmente la tesi di Giacomo Sangalli (1821-97) ${ }^{8}$, professore di anatomia patologica a Pavia, il quale affermò nel 1866 di «avere alcuni anni trovato l'anchilostoma pressochè nella metà dei cadaveri, che venivano sezionati nella mia scuola ${ }^{9}{ }^{9}$ : e questa stessa tesi il Sangalli ${ }^{10}$ ribadì un decennio più tardi, nel 1876.

8 G. Sangalli, Geografia elmintologica: Anchilostoma e trichina, Giornale di anatomia e fisiologia patologica 3 (1866) 100-106.

9 «Più spesso che nel duodeno lo incontrai nelle prime anse del digiuno, dove giaceva appiccicato alla mucosa mediante denso ed abbondante muco grigiastro, che sempre si osserva in questo genere d'elmintiasi»: semplicemente appiccicato alla mucosa, e non infisso in questa con gli uncini periorali, come lo aveva trovato il Bilharz nei clorotici egiziani. «Bilharz ai tristi effetti di questa emorragia attribuisce la clorosi, che in Egitto è tanto frequente. Fra noi questo fenomeno non si è ancora osservato per effetto dell'anchilostoma, ma potei convincermi della sua possibilità; invero parecchie volte il verme aderisce lassamente per la sua estremità anteriore alla mucosa; qualche volta, discostandonelo, vi si trova una macchietta rossiccia, che è sangue effuso nel tessuto della mucosa per effetto della puntura del verme; una volta trovai una larga ecchimosi sulla mucosa, e nel mezzo l'anchilostoma saldamente infitto per la bocca.»

10 G. SANGaLLI, Sopra alcuni punti controversi di elmintologia, Mem. d. R. Istit. Lombardo (Classe di scienze matem. e natur.) 13 (1876) 349-61: «... non so assegnare una ragione plausibile» al fatto «importante, che il verme, avendo la bocca armata di quattro uncinetti tanto in Egitto che in Italia, in quel paese (dove è frequentissimo) spieghi la funesta proprietà d'infiggere il suo capo nella mucosa dell'intestino in cui si annida, procurando per tal modo emorragie, principj di mali gravi; mentre presso di noi non ha che eccezionalmente un simile costume, e quindi riesce quasi innocuo.» $\grave{E}$ qui opportuno precisare che, con quel «quasi innocuo», il Sangalli intende riferirsi al caso di un uomo di 57 anni profondamente anemizzato che risultò, al tavolo anatomico, portatore di 700 anchilostomi, senza peraltro che fossero reperibili trafitture, recenti e antiche, della mucosa intestinale: "pure confesso che l'anemia riscontrata in quel cadavere, e con essa la causa della morte del paziente, non mi sarebbero ben chiare, se non ammettessi una qualsiasi funesta influenza di quel verme sull'intestino, onde poi la cattiva chilificazione e sanguificazione. » In qualche raro caso, quindi, sui molti in cui viene ospitato 
Altrettanto aggressivo quanto l'egiziano è invece l'Ankylostoma brasiliano, e col suo sanguisugio provoca la c. d. anemia intertropicale o clorosi dei tropici: in questi casi il reperto autoptico è sovrapponibile a quello della clorosi egiziana, come costatò a Bahia, fin dal 1865, Otto Wucherer (1820 a 1873$)^{11}$.

Il 20 maggio 1877, Prospero Sonsino ${ }^{12}$, un medico fiorentino attivo in Egitto, comunicò alla Società medico-fisica Fiorentina le osservazioni autoptiche da lui compiute al Cairo sulla clorosi da Ankylostoma secondo la tesi del Griesinger: e il 30 settembre dello stesso anno, Carlo Morelli ${ }^{13}$, influenzato dal Sonsino, ritrovò a Firenze l'Ankylostoma nel cadavere di una donna morta di «anemia perniciosa progressiva», inaugurando così in Italia la fase dei ritrovamenti extralombardi dell'Ankylostoma.

Giova però ricordare che siamo ormai entrati nel periodo cruciale della conquista del contagio vivo. Le celebri scoperte di Louis Pasteur (1822-95) e di Robert Koch (1843-1910) dimostrano che minutissimi esseri viventi provocano gravi malattie degli animali superiori e dell'uomo. Il 1876 - l'anno del fondamentale lavoro del Koch sul bacillo del carbonchio - segna una svolta decisiva anche nello studio biologico dell'Ankylostoma. Nel secondo volume, proprio allora apparso, del trattato Die menschlichen Parasiten und die von ihnen herrührenden Krankheiten Rudolf Leuckart (1823-98) ${ }^{14}$ imposta lo studio biologico dell'Ankylostoma. Data l'irreperibilità del verme umano in Germania, il Leuckart porta le sue ricerche

nell'organismo umano, l'Ankylostoma esplica un'azione anemizzante, che però non è riferibile a sanguisugio, bensì all'alterato assorbimento intestinale. Sappiamo che quanto più lungo è il tempo intercorso fra la morte e l'apertura del cadavere, tanto più numerosi sono i vermi distaccatisi dalla mucosa e liberi nel lume intestinale: e in base a questa costatazione spieghiamo quella che fu allora interpretata come une diversa aggressività ematofagica fra Ankylostoma italiano ed egiziano.

Del Sangalli si vedano anche le Annotazioni critiche sull'anchilostoma duodenale, Rendiconti d. R. Istit. Lombardo 11 (1878) 460-467.

11 O. Wucherer, Über die Anchylostomenkrankheit, tropische Chlorose oder tropische Hypoämie, Deutsches Archiv für klinische Medicin 10 (1872) 379-400. - L'importanza delle ricerche del Wucherer era stata subito messa in rilievo nel lavoro di W. GRIESINGER, Das Wesen der tropischen Chlorose, Archiv der Heilkunde 7 (1866) 381.

12 P.Sonsino, L'Anchilostoma duodenale in relazione coll'anemia progressiva perniciosa, L'Imparziale 18 (1878) 227-234.

13 C.Morelli, Intorno ad un caso d'anemia progressiva con Anchilostoma duodenale, Lo Sperimentale 41 (1878) 27-39.

14 R.Leuckart, Die menschlichen Parasiten und die von ihnen herrührenden Krankheiten 2, Leipzig/Heidelberg 1876, 410-460 e 480. 
sul Dochmius trigonocephalus del cane, seguendone accuratamente lo sviluppo: formazione dell'embrione, schiusa dell'uovo, liberazione della larva e successive mute fino all'animale perfetto.

Affine al Dochmius del cane è il Dochmius Balsami ${ }^{15}$ del gatto, scoperto nel settembre del 1876 da Battista Grassi (1854-1925) che, al termine del IV anno di medicina a Pavia, trascorreva le vacanze estive nel nativo paese di Rovellasca (prov. di Como). Rientrato nell'autunno a Pavia, il Grassi continuò lo studio del verme nel laboratorio di anatomia comparata, diretto da Leopoldo Maggi (1840-1905), associando alle ricerche l'assistente Corrado Parona (1848-1922) ${ }^{16}$ ed estendendole all'Ankylostoma duodenale. In un breve articolo apparso sulla Gazzetta Medica ${ }^{17}$ del 18 maggio 1878 i due autori facevano il punto dei fatti da loro scoperti sulla embriologia del verme.

L'uovo dell'Ankylostoma, sia nel corpo della femmina, sia libero nel contenuto intestinale, era stato raffigurato nelle tavole stesse del Dubini, il quale non aveva peraltro sfruttato questa sua osservazione. Il Grassi e C.Parona costatarono innanzitutto, con ricerche anatomiche sistematiche, che la presenza del verme nell'intestino umano è sempre associata alla presenza di uova in vari stadi di segmentazione fino alla morula. Anche nelle deiezioni fresche e nel vomito appena emesso di portatori sono sempre reperibili «uova in stadio di segmentazione (non mai ad uno stadio oltre

15 In onore di Giuseppe Balsamo-Crivelli (1800-1874), professore di storia naturale e, infine, di zoologia e anatomia comparata all'Università di Pavia. Il Balsamo-Crivelli continuò gli studi di Agostino Bassi (1773-1856) sulla crittogama che produce il mal del calcino del baco da seta e la identificò in una nuova specie del genere Botrytis, che dapprima denominò paradoxa e poi Bassiana.

16 Di una nuova specie di Dochmius (Dochmius Balsami). Nota del dott. Corrado Parona e di Battista Grassi, studente del $5^{\circ}$ anno di medicina; presentata dal M.E. professor Giacomo Sangalli, Rendiconti d. R. Istit. Lombardo 10 (1877) 190-195 e 1 tav. f.t. (tornata del 15 marzo 1877). - L. MAGgi, Sugli studj di C. Parona e G. B. Grassi intorno all'Anchilostoma duodenale Dub., Rendiconti d. R. Istit. Lombardo 11 (1878) 428-436. Seguono a pp. 436-438 le osservazioni del S.C. prof. P.PAvesi e, infine, a pp. 438-439: G.SANGALLI, Contro osservazioni alle osservazioni del Prof. P. Pavesi alla lettura del professor Maggi.

17 Intorno all'anchilostoma duodenale (Dubini). Annotazioni di Battista Grassi, laureando in medicina, Corrado dott. Parona e Ernesto dott. Parona, Gazz. med. Ital. - Lombardia 5 d. s. VII (1878) 193-196.

Intorno all'anchilostomiasi. Osservazioni dei dottori Grassi Battista e Parona Ernesto, con un'Appendice embriologica, dello stesso dott. Grassi e dott. Corrado Parona, Ann. universali di med. e chir. 247 (1879) 407-424 e 1 tav. 
la morula) ...»: e queste uova sono facilmente distinguibili da quelle di altri elminti parassiti. Emesse dall'organismo umano, le uova proseguono il loro sviluppo: e i due ricercatori osservarono la formazione dell'embrione, la schiusa e la fuoriuscita della larva, che, cambiata «la pelle almeno due volte», si trasforma in un'altra larva, che subisce una nuova muta. A complemento di queste ricerche, il Grassi trangugiò - e impunemente - «una grossa pillola carica di uova di anchilostoma in via di segmentazione».

Queste ricerche del Grassi e di C.Parona sono altrettanto fondamentali dal punto di vista biologico e da quello clinico, poichè indicarono nell'esame microscopico delle feci il mezzo per riconoscere la presenza del verme nell'uomo vivente ${ }^{18}$ : mezzo che il Grassi si mise immediatamente a sfruttare, associandosi, questa volta, a Ernesto Parona (1849-1902) ${ }^{19}$, fratello di Corrado, che fungeva da aiuto alla Clinica Medica dell'Università di Pavia, diretta da Francesco Orsi (1828-1900).

Grazie all'esame delle feci, i due giovani ricercatori riuscirono a riconoscere la presenza del verme in alcuni «casi di gravi anemie» ad etiologia «oscura e insufficiente» ricoverati nella clinica; e osservarono che il verme adulto, mentre è regolarmente assente nelle feci degli infestati, viene invece espulso per effetto della somministrazione di un antielmintico - polvere composta di santonina, calomelano e resina di gialappa - per cui ritennero «non ... troppo ardita la speranza di una efficace medicazione».

In un caso l'autopsia non tardò a confermare la diagnosi di anemia da Ankylostoma formulata «in extremis» dal giovane studente e accolta con ironico scetticismo dall'illustre clinico di Pavia ${ }^{20}$.

«Anche in Italia », quindi «avviene nell'uomo la così detta clorosi d'Egitto, perchè l'anchilostoma pur nel nostro paese succhia sangue»: affermò il Grassi $^{21}$ in un lavoro del successivo novembre 1878.

18 «La diagnosi di anchilostoma è facilissima; a raggiungere prontamente tale meta basta osservare ad un ingrandimento microscopico almeno di 90 diametri un po' di fece o di vomito diluito con un mestruo qualunque. Se la materia è recente, troveremo appena le uova d'anchilostoma in segmentazione; se è stantia, anche gli embrioni e le larve.»

19 Dr. Ernesto Parona (1849-1902). Discorsi Grocco-Maroni-Brocca. Inaugurando Ricordo Perenne nell'Ospedale Fate-Bene-Fratelli. Milano 30 maggio 1904, Novara 1905.

20 L'aneddoto è riferito da Filippo Silvestri nell'opuscolo miscellaneo Onoranze a Battista Grassi, Roma 1925,33-34, ed è integralmente riportato a pp. 117-118 del primo lavoro di L. Belloni 1962 qui citato alla nota 1.

21 B.Grassi, Intorno ad una nuova malattia del gatto, analoga alla clorosi d'Egitto (anemia da anchilostomi) dell'uomo, Gazz. med. Ital. - Lombardia 5 d. s. VII (1878) 451-454. 
I suoi lavori coi fratelli Parona erano stati in proposito fondamentali, e andavano perfezionati con la prova terapeutica: la sola che avrebbe assicurato il rapporto etiologico fra Ankylostoma e anemia: bisognava cioè guarire l'anemia somministrando un antielmintico che liberasse il paziente dall'Ankylostoma. Nelle mani di Giovan Cosimo Bonomo (1687) la medicazione topica ${ }^{22}$, intesa ad uccidere l'acaro, aveva avuto una parte importante per affermare l'etiologia acarica della scabbia ${ }^{23}$.

A Torino, la ricerca dei portatori di Ankylostoma fu iniziata da Bonaventura Graziadei (1852-1935) ${ }^{24}$, il quale praticò l'esame copromicroscopico a malati degenti in diversi reparti, ospedalieri e universitari, e in particolare nelle due Cliniche mediche - generale e propedeutica - rispettivamente dirette da Luigi Concato (1825-82) e da Camillo Bozzolo (1845-1920).

Nel giugno del 1879 due casi individuati dal Graziadei - entrambi fornaciai da mattoni, quali erano stati in buona parte i pazienti della casistica del Grassi e di E.Parona - fornirono lo spunto al Bozzolo per concludere il corso di clinica medica propedeutica con una lezione dal titolo impegnativo: L'anchilostomiasi e l'anemia che ne conseguita (anchilostomanemia) ${ }^{25}$. In questa lezione il Bozzolo, oltre all'importanza delle ricerche del Grassi e dei fratelli Parona, mise in risalto anche un esame di laboratorio che si sarebbe rivelato tosto prezioso per valutare nelle sue oscillazioni il grado dell'anemia: il dosaggio dell'emoglobina col cromocitometro proprio allora messo a punto da Giulio Bizzozero (1846-1901) ${ }^{26}$. La sperimentazione terapeutica fallì anche nei due casi del Bozzolo: «... noi non conosciamo alcun mezzo capace di rendere inattivi, di uccidere, di espellere gli anchilostomi e invanamente abbiamo adoperato nella clinica quasi tutti gli antielmintici.» Oltre al Graziadei, quale copromicroscopista, e a Ignazio Fenoglio,

${ }^{22}$ L. Belloni, La medicazione topica nella scoperta della etiologia acarica della scabbia, Simposi Clinici 1 (1964) XXI-XXVI; I secoli italiani della dottrina del contagio vivo, Simposi Clinici 4. (1967), XLIX-LVI, 5 (1968) I-VIII.

${ }^{23}$ Che costituì un perspicuo modello al quale fecero ripetutamente riferimento i sostenitori del contagionismo. Così, ad es., il Bilharz si chiede «ob unser Distomum Haematobium zur Dysenterie in derselben Beziehung steht, wie Acarus scabiei zur Krätze» (cf. p. 76 del lavoro qui citato alla nota 7).

24 N. Valobra, Bonaventura Graziadei (1852-1935), Giornale della R. Accademia di Medicina di Torino (Parte II: Memorie originali) 49 (1936) 24-35.

${ }^{25}$ Giornale internazionale delle scienze mediche 1 (1879) 1054-1069 e 1245-1253.

${ }^{26}$ G. Bizzozero, Il cromo-citometro. Nuovo strumento per dosare l'emoglobina del sangue, Atti d. R.Accademia delle Scienze di Torino 14 (1878-1879), 899-942 e tav. XII. La nota del Bizzozero fu presentata da M. Lessona nell'adunanza dell'11 maggio 1879. 
quale cromocitometrista, troviamo ricordato, nella lezione del Bozzolo, anche uno studioso che contribuì alla misurazione delle uova di Ankylostoma: Edoardo Perroncito (1847-1936), patologo alla Scuola Veterinaria di Torino.

Il terreno era ormai dissodato per la soluzione dell'enigma patologico del San Gottardo, che andava tragicamente profilandosi. I lavori del traforo, inaugurati nel 1872, volgevano ormai al termine; ma si sussurrava che fosse comparsa endemica fra quegli operai una malattia cachettizzante che da tempi remoti veniva considerata triste appannaggio degli addetti ai lavori minerari: la c.d. cachessia dei minatori. Resi inabili al lavoro, molti minatori malati tornavano alle loro case, e ricorrevano agli ospedali locali. Minatori cachettici reduci dal traforo furono ricoverati anche alla Clinica medica generale di Torino, diretta dal Concato, e sottoposti ad esame copromicroscopico dal Perroncito ${ }^{27}$, risultarono portatori di Ankylostomi.

Nel febbraio del 1880 questa diagnosi clinica ricevette la conferma del tavolo anatomico. Si trattava di un minatore gravemente cachettico nel quale la trasfusione intraperitoneale di sangue defibrinato ${ }^{28}$, tentata «in extremis», aveva scatenato una peritonite siero-fibrinosa rapidamente mortale. All'autopsia, solennemente praticata di fronte ad autorevole e folto pubblico dal professore di anatomia patologica, Francesco-Vittorio Colomiatti (1848-83), «si trovarono nel duodeno e nel digiuno oltre 1500 anchilostomi, parecchi dei quali mostravano evidentissima la loro proprietà ematofaga: il loro intestino conteneva una quantità varia di emasie ottimamente conservate. ${ }^{29}$

Forte dei primi successi, il Perroncito sviluppò le ricerche, sempre più convincendosi che l'anemia del Gottardo si dovesse sostanzialmente imputare alla infestazione verminosa, e in prima linea all'Ankylostoma (ammettendo anche una partecipazione, variabile da caso a caso, della Anguillula intestinalis e stercoralis, scoperta dal Normand in Cocincina nel 1877, e ritrovata nel settembre del 1878 dal Grassi e dai fratelli Parona a Pavia).

27 E. Perroncito, Osservazioni elmintologiche relative alla malattia sviluppatasi endemica negli operai del Gottardo, R.Accad. dei Lincei. Mem. d. Classe di scienze fisiche, matem. e natur. 7 (1880) 1-55 e 1 tav. - La malattia dei minatori dal S. Gottardo al Sempione. Una questione risolta, Torino 1909. - La maladie des mineurs du St.Gothard au Simplon. Une question résolue. Appendice, Turin 1912.

${ }^{28}$ LAVA, La trasfusione del sangue per la cavità del peritoneo, L'Osservatore, Gazzetta delle Cliniche. Organo uff. d. Soc. di med. e chir. di Torino 16 (1880) 81-82.

${ }^{29}$ Pag. 3 del lavoro di E.Perroncito 1880 qui citato alla nota 27. 
Il Perroncito compì inoltre fondamentali ricerche in vitro sulle metamorfosi larvali dell'Ankylostoma e le condizioni ottimali per lo sviluppo larvale. La necessità di un periodo di vita libera allo stato larvale nelle feci eliminate, e le condizioni di ambiente ottimali a questo fine - terreno umido! - spiegano la diffusione endemica del verme entro determinati aggregati umani (contadini, fornaciai da mattoni, minatori) e indicano pertanto la strada che deve essere battuta dalla profilassi.

Il Perroncito saggiò, inoltre, l'azione di svariati antielmintici sulle larve di Ankylostoma maturate in vitro, e concluse per la preminenza del felce maschio, già noto da tempo come tenifugo. Questo farmaco venne infatti favorevolmente sperimentato su minatori degenti nella clinica del Concato: ed altri ricercatori lo seguirono sulla stessa via: in prima linea, Ernesto Parona, che, dopo iniziali tentennamenti e insuccessi, mise a punto lo schema terapeutico ottimale e lo collaudò - come vedremo - con larghissima casistica.

Accanto al felce machio, altro antielmintico specifico fu contemporaneamente trovato nel timolo ${ }^{30}$, per merito del Bozzolo.

La soluzione del problema terapeutico significò al tempo stesso la vittoria della dottrina contagionista, sostenuta del Perroncito, rispetto alla teoria localista, cui aderivano invece l'igienista Luigi Pagliani (1847-1932) e lo stesso Bozzolo, i quali, dopo aver portato le loro ricerche nel vivo stesso dei cantieri, ossia per l'appunto «in loco», esposero le loro conclusioni in una celebre seduta della R. Società Italiana di Igiene svolta a Milano il 10 aprile $1880^{31}$.

I due autori avevano ovviamente riscontrato l'Ankylostoma nei minatori cachettici, ma, anzichè attribuire interamente a suo carico la malattia, lo consideravano piuttosto come un fattore morbigeno complementare, venuto «ad aggravare di molto le condizioni già tristi del tunnel».

Per rendere facilmente comprensibile la posizione della coppia BozzoloPagliani, ricordo, sia pur di sfuggita, che ci troviamo alla viglia dei lavori di Robert Koch sul vibrione del colera, che fecero prevalere, per questa malattia, la dottrina contagionista rispetto alla teoria localista, sostenuta,

30 Ampia casistica terapeutica a favore del timolo fu prodotta da B. GraZiadeI, Il Timolo nella cura dell'anchilostomanemia, Giorn. d. R. Accad. di medicina di Torino 30 (1882) 821-855.

${ }^{31}$ L. Pagliani e C.Bozzolo, L'anemia al traforo del Gottardo dal punto di vista igienico e clinico, Giorn. d. Soc. Ital. d'Igiene 2 (1880) 276-34.6. 
invece, da Max Pettenkofer (1818-1901), il fondatore della igiene moderna. Il colera - che decimò con ripetute ondate l'Europa a partire dagli anni trenta dell'Ottocento - dipendeva, secondo il Pettenkofer, da una disposizione locale cronologicamente condizionata, da una costellazione di fattori ambientali, che reciprocamente confluiscono soltanto in determinati luoghi e in determinati tempi: e confluendo esercitano una influenza colerigena sulle popolazioni (epi-demia). E' compito della nuova igiene, secondo il Pettenkofer, sfruttare i metodi della chimica e della fisica per valutare in termini numerici questi diversi fattori ambientali, i caratteri del suolo, delle acque sotterranee, e così via: in breve, Le arie, le acque e $i$ luoghi, per ricordare il titolo di un celeberrimo trattato d'Ippocrate.

Nella profondità del tunnel convergeva un complesso di fattori ambientali indubbiamente abnormi: agglomerazione di operai, difetto di ventilazione, ristagno dei gas delle mine e in particolare della polvere di dinamite scoppiata, accumulo di materiali escrementizi, mancanza di luce, aumento di temperatura, e così via: e ciascuno di questi fattori era andato aggravandosi man mano procedevano i lavori del tunnel, spiegando le preoccupazioni del Bozzolo e del Pagliani di fronte a tali «condizioni che solo in questi ultimi anni si verificarono, essendo questa una delle prime volte che l'uomo sia penetrato così addentro nelle viscere della terra, portando con titanico ardimento lo scalpello demolitore nel seno della roccia alla profondità di oltre settemila metri » : e queste preoccupazioni rispecchiano le attuali nostre per le condizioni ambientali, in parte ignote, alle quali vengono esposti i cosmonauti.

Il dibattito fra contagionismo e localismo fu rapidamente messo a tacere dai brillanti risultati della terapia antielmintica specifica, che, come si è detto, potè in breve tempo avvalersi di due farmaci di grande efficacia: il felce maschio e il timolo, per merito del Perroncito e, rispettivamente, del Bozzolo. La malattia non migliora, nè guarisce sottraendo il minatore cachettico alle condizioni ambientali del tunnel; ma soltanto liberandolo dall'Ankylostoma: e il processo di guarigione è documentabile anche in termini numerici dosando l'emoglobina col cromocitometro del Bizzozero.

Ernesto Parona - che avevamo lasciato alla Clinica Medica di Pavia intento a collaborare col Grassi - fu chiamato nel novembre del 1879 alla direzione del Civico ospedale di Varese; e in questa carica, che tenne fino al settembre del 1885, applicò la sua esperienza di pioniere per consolidare l'etiologia elmintica dell'anemia del Gottardo e metterne a punto la terapia, tanto più che l'ospedale da lui diretto fu uno dei prescelti per la cura 
degli ex-minatori cachettici a carico del governo. Nella Relazione intorno alla cura dei minatori del Gottardo accolti a carico del R. Governo nel Civico Ospedale di Varese, licenziata nell'agosto del 1884 e stampata a Varese nel 1885, il Parona poteva produrre una casistica ${ }^{32}$, anche laboristicamente impeccabile, che comprendeva 249 ex-minatori cachettici dimessi completamente guariti grazie al trattamento antielmintico, praticato soprattutto col felce maschio.

Risolto il problema dell'anemia del Gottardo - con le rilevanti implicazioni di ordine profilattico e terapeutico che esso comportava - veniva sul tappeto il problema più generale dell'anemia dei minatori addetti a miniere permanenti. Nel dibattito fra contagionismo e localismo, la vittoria arrise al primo; ma non fu una vittoria fulminea, e dovette progressivamente conquistare, dopo il Gottardo, miniera per miniera. Tra le prime conquiste figurano le solfatare di Lercara Friddi (prov. di Palermo), grazie all'opera di Alfonso Giordano (1842-1915) ${ }^{33}$, che, riconosciuta la presenza dell'Ankylostoma in minatori anemici ${ }^{34}$, già nel febbraio del 1882 potè produrre alcuni casi guariti col solo impiego del felce maschio ${ }^{35}$.

La soluzione dell'enigma patologico del San Gottardo fu una conquista della scienza che ebbe grande risonanza umanitaria e sociale, soprattutto dopo che il suo enorme valore pratico fu collaudato circa un ventennio più tardi, quando, ammaestrato dalla tragedia del Gottardo, l'uomo potè strappare alla natura una nuova, grande vittoria - il traforo del Sempione senza aver dovuto versare il minimo pedaggio all'Ankylostoma. Attivo in prima linea fu, questa volta, il medico piemontese Giuseppe Volante (1870 a 1936), «sanitario dell'impresa dal lato sud».

${ }^{32}$ Per i primi casi studiati da Ernesto Parona all'Ospedale di Varese cf.: E.Parona, L'anchilostomiasi e la malattia dei minatori del Gottardo, Annali universali di med. $e$ chir. 253 (1880) 177-202 e 464.

${ }^{33} \mathrm{Nel}$ cinquantenario della morte, la figura del Giordano è stata commemorata all'Università di Palermo il 28 Giugno 1965 ad opera di Giovanni Fradà e di Giuseppe Pontieri. Cfr. Folia medica 48 (1965) fasc. 10.

${ }^{34}$ E.Parona, L'anchilostomiasi nelle zolfare di Sicilia, Annali universali di med.e chir. 277 (1886) 464-468.

35 Giorn. d. R. Accad. di med. di Torino 30 (1882) 90: "Il socio Perroncito a nome del sig. Dott. Giordano di Lercara, annuncia come questi abbia trovato nelle solfatare in individui, addetti alle miniere, affetti da anemia l'anchilostoma, e come curato l'anchilostoma mercè il felce maschio l'anemia guarisce.» (Dal verbale della seduta del 24 febbraio 1882.) 
Il traforo del Sempione fu celebrato a Milano nel 1906 con una serie imponente di manifestazioni, culminate nel $« \mathrm{I}^{0}$ Congresso Internazionale per le Malattie del Lavoro », mentre, ad opera di Luigi Devoto (1864-1936), era in gestazione la «Clinica del Lavoro», inaugurata il 20 marzo 1910.

Frutto di quel Congresso fu la istituzione della «Commissione Internazionale per le malattie professionali» avente a segretario generale Luigi Carozzi, un giovane allievo del Devoto, che passerà nel 1921 al «Bureau International du Travail» presso la Società delle Nazioni a Ginevra.

All'imbocco meridionale della galleria del Gottardo la replica di un celebre rilievo in bronzo alimenta, a partire dal 1932, il ricordo del tributo di sangue imposto all'uomo dalla grande impresa. Si intitola Le vittime del lavoro ed è opera di Vincenzo Vela (1822-91). Anche se l'artista sembra volutamente polarizzarci verso l'incidente traumatico, non dimentichiamo che il grande tributo di sangue fu invece quello versato all'Ankylostoma: e fu versato dai veri protagonisti di questa storia: dai minatori che, aprendo col loro lavoro e il loro sacrificio una breccia nella barriera alpina, hanno avvicinato i popoli d'Europa e pertanto contribuito alla loro unione. 